\title{
Preparation of a Rigid and nearly Coplanar Bis-Tetracene Dimer through Application of the CANAL Reaction
}

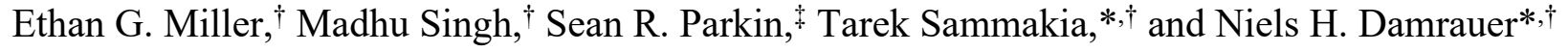 \\ 'Department of Chemistry, University of Colorado Boulder, Boulder, Colorado 80309, United States \\ Department of Chemistry, University of Kentucky, Lexington, Kentucky 40506, United States
}

\begin{abstract}
A rigid tetracene dimer with a substantial interchromophore distance has been prepared through the application of the recently developed catalytic arene-norbornene annulation (CANAL) reaction. An iterative cycloaddition route was found to be unsuccessful, and so a shorter route was adopted whereby fragments were coupled in the penultimate step to form $13: 1$ mixture of two diastereomers, the major of which was isolated and crystallized. Constituent tetracene moieties are linked with a rigid, well-defined bridge, and feature a near co-planar mutual orientation of the acenes.
\end{abstract}

Anthropogenic climate change has fueled research in next-generation solar energy conversion technologies including ones aiming to bypass the Shockley-Queisser limit, the theoretical ceiling on the power-conversion efficiency of single-junction solar cells. ${ }^{1,2}$ A centerpiece in these efforts is the study of Singlet Fission (SF), a phenomenon of energetic proportionation between an excited-state chromophore and a ground-state neighbor. ${ }^{3}$ The SF reaction originates in a single chromophore, or material-extended, singlet exciton excited state that is generally the lowest energy, $\mathrm{S}_{1}$. The reaction culminates in the formation of independent triplets on separate chromophores, first passing through a multiexcitonic state consisting of two triplets coupled into a net singlet ( $\left.{ }^{1} \mathrm{TT}\right)$. It is the study of factors controlling $\mathrm{S}_{1} \rightarrow{ }^{1} \mathrm{TT}$ dynamics that led us and others to consider molecular dimers as fundamental platforms where reaction parameters such as driving force and interchromophore electronic coupling could be systematically varied, drawing on a vast toolbox of organic synthesis to control both bridge and chromophore type and the nature of connectivity. ${ }^{4-13}$ A central theme in our efforts has been the consideration of structurally well-defined acene dimers using bicyclic alkyl bridging units derived from norbornadiene ${ }^{14-22}$ including synthesis specific work..$^{18,20}$

Recently, it has been realized that dynamics at play in the initial steps of SF may have value well beyond solar technologies, reaching into quantum information sciences (QIS) ${ }^{23}$ In that field, qubits (quantum bits) are needed for computation, requiring the initialization of quantum-mechanically pure states, for example $\alpha$ versus $\beta$ spin states in a magnetic field that can function as a 1 or 0 in logic gates. ${ }^{24}$ In order to avoid the need for exceedingly low temperatures during initialization and computation (currently a requirement for stateof-the-art quantum computers), there is great interest in identifying systems where optical excitation is coupled with spin selectivity in the dynamics resulting in the generation of pure states ${ }^{25-27}$ that can then be subjected to subsequent operations needed for computation, 
using external radio-frequency fields along the lines of what is employed in NMR or EPR spectrometers. The reasons that SF becomes interesting in this context is rooted in two main ideas. The first is tied to the idea that in excited-state manifolds comprised of two coupled triplets, the ${ }^{1} \mathrm{TT}$ is not the only multiexciton state. One needs to also consider the ${ }^{3} \mathrm{TT}$ and the ${ }^{5} \mathrm{TT}$, and unlike the ${ }^{1} \mathrm{TT}$, both of these are subjected to degeneracy-lifting Zeeman interactions in a magnetic field, thereby energetically isolating the $\mathrm{M}_{\mathrm{s}}$ magnetic sublevels (the ${ }^{3} \mathrm{TT}$ becomes ${ }^{3} \mathrm{TT}_{1},{ }^{3} \mathrm{TT}_{0}$, and ${ }^{3} \mathrm{TT}_{-1}$ while the ${ }^{5} \mathrm{TT}$ becomes the ${ }^{5} \mathrm{TT}_{2},{ }^{5} \mathrm{TT}_{1},{ }^{5} \mathrm{TT}_{0},{ }^{5} \mathrm{TT}_{-1}$, and ${ }^{5} \mathrm{TT}_{-2}$ ). It is pairs of these sublevels that may be able to function as qubits in the same way as described above with $\alpha$ versus $\beta$ spin states, although there is an expectation that the ${ }^{5} \mathrm{TT}_{0}$ form preferentially from the ${ }^{1} \mathrm{TT}$ following spin evolution ${ }^{28,29}$ and that this can serve as an initialized qubit. ${ }^{23}$ The second idea is more specifically tied to the synthetic goals in this paper. It has recently been predicted that when two chromophores share a common set of axes relative to an external magnetic field - in other words when they are parallel - selection rules are imposed such that dynamics leading to loss of the ${ }^{5} \mathrm{TT}_{0}$ to other states in the manifold will be slowed. ${ }^{23}$ These are then conditions that could enable initialization of a quantum mechanically pure state (the qubit) as well as time needed to perform qubit operations.

As noted above, our groups have been interested in the synthesis and study of rigid acene dimers and we have previously utilized [2.2.1] bicyclic bridges that impose structural definition via two covalent points of attachment between the bridge and each chromophore. A computational model of an acetylene-substituted pentacene dimer (BP1') leads to a prediction of $113^{\circ}$ for the interchromophore angle, a significant deviation from coplanarity (Fig. 1 (top) and a related tetracene system ${ }^{14}$ ). As a follow up to these studies, we have targeted extended ("X") tetracene and pentacene analogues of BT1' and BP1' (TIPS-BTX and TIPS-BPX, respectively, Fig. 1 (bottom)). In these structures, the acene chromophores are fused to norbornyl groups via cyclobutenyl bridging units connected by a central aryl group. The cyclobutenyl groups ensure a rigid core and the fusion to the norbornyl groups stabilize this functionality to ring opening, ${ }^{30}$ which would result in an anti-Bredt olefin. There are two diastereomers of the dimers that are possible via our synthesis (vide infra) wherein the constituent chromophores are syn- or anti-, and computational models of pentacene versions - again with acetylene substituents - are shown in Fig. 1 (top, syn-BPX and anti-BPX). In both isomers, the chromophores are predicted to have near-perfect coplanarity with an interchromophore angle of $179^{\circ}$ for the anti-isomer, and $175^{\circ}$ for the syn-isomer. Although both isomers have the desired near coplanar arrangement of chromophores, a mixture would introduce complexity in future interpretation of excited state dynamics and spin dynamics and it is therefore important to prepare and isolate these compounds as single diastereomers, not mixtures. In this manuscript we discuss the synthesis of TIPS-BTX and our initial unsuccessful approach to TIPS-BPX. We will report on the synthesis of TIP-BPX shortly. 


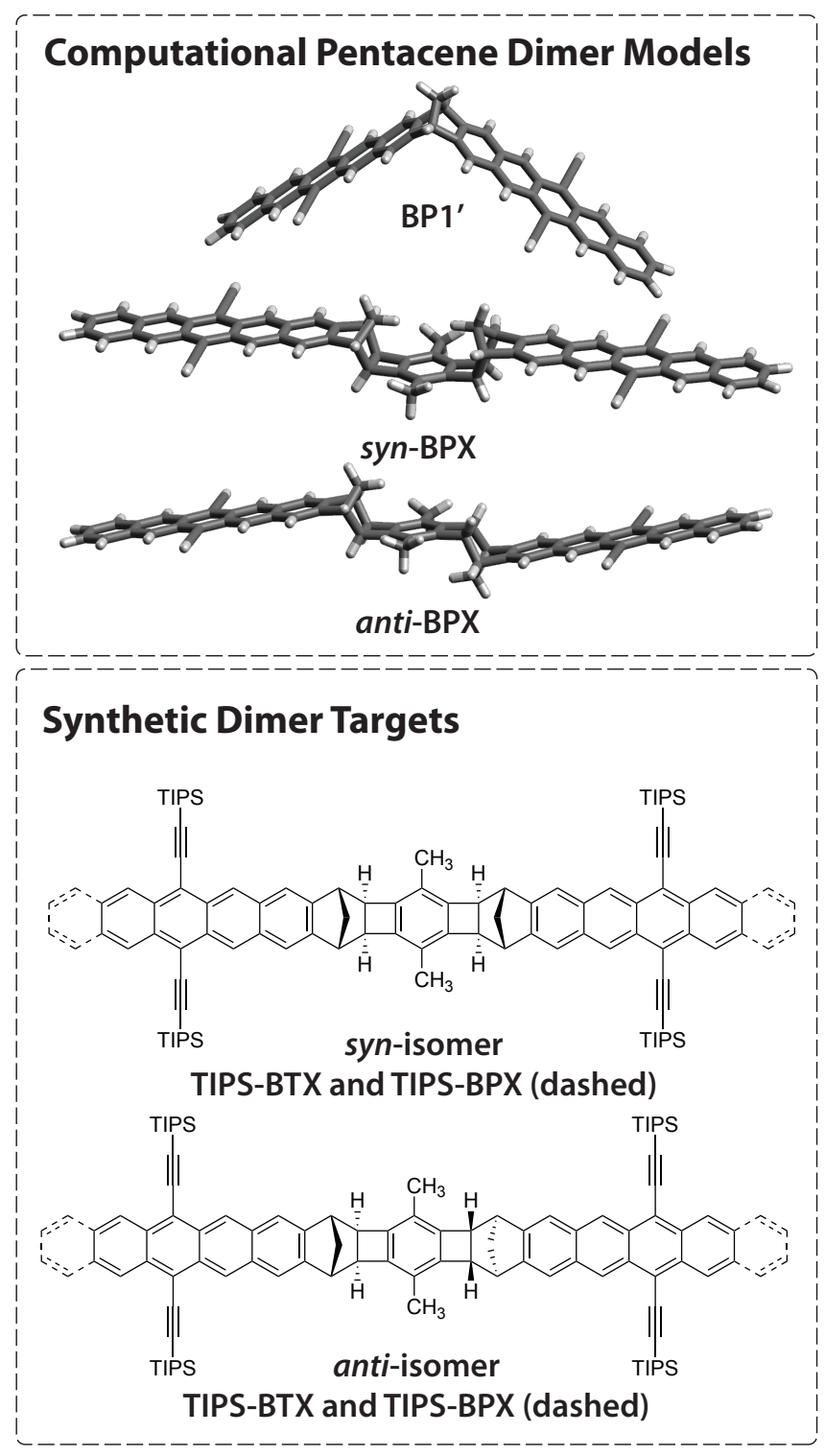

Figure 1. (Top) Computational models of acetylene-substituted pentacene dimers. Density functional theory (DFT) was used with the $\omega$ B97X-D density functional, the 6-31G(d) basis set, and a polarizable continuum model of solvent parameterized for toluene. The calculated interchromophore angle of $\mathrm{BP} 1^{\prime}$ is $113^{\circ}$, syn-BPX is $175^{\circ}$, and anti-BPX is $179^{\circ}$. (Bottom) Dimeric targets TIPS-BTX, TIPS-BPX (dashed bonds) with both possible isomers syn and anti shown.

Initial Synthetic Approach. Our initial retrosynthetic analysis of TIPS-BPX drew from our experience with the synthesis of TIPS-BP1 ${ }^{\prime 20}$ and is shown in Scheme 1. As in the case of our prior synthesis, we wished to employ a 2-directional Diels-Alder approach between a bis-diene in the central portion of the molecule and a quinone dienophile. Ideally, this would be a one-pot, double-DielsAlder reaction, though a sequential approach as was employed in our synthesis of TIPS-BP1' was considered as a backup. In either case, the product of the Diels-Alder reaction would be subjected to a sequence consisting of oxidative aromatization $\left(\mathrm{O}_{2} / \mathrm{Al}_{2} \mathrm{O}_{3}\right)$ of the cycloadduct followed by reduction of the quinone $\left(\mathrm{NaBH}_{4}\right)$ to the corresponding diol and a final reductive aromatization $\left(\mathrm{SnCl}_{2}\right)$ to provide the bis-acene. 


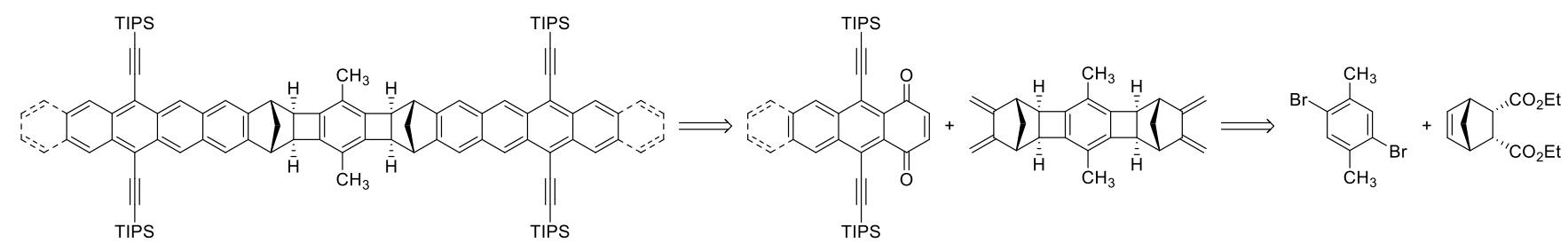

Scheme 1. Initial retrosynthesis of TIPS-BTX, TIPS-BPX (dashed bonds).

We adopted a transform-goal approach to the synthesis of bis-diene 5 (Scheme 2) using the catalytic arene-norbornene annulation (CANAL) reaction developed by $\mathrm{Xia}^{31}$ for the construction of the cyclobutenyl fragments. The CANAL transformation typically employs a bromo-arene, wherein one of the sites ortho- to the bromine is blocked to direct reactivity to the other site. As such, we chose the di-bromo $p$-xylene derivative $\mathbf{1}$ as our starting material, which we subjected to the CANAL reaction with norbornene bis-ester 2 $\left(\mathrm{Pd}(\mathrm{OAc})_{2} / \mathrm{PPh}_{3} / \mathrm{Cs}_{2} \mathrm{CO}_{3}, 82 \%\right)$ to provide tetraester 3 as a 4:1 mixture of two exo-stereoisomers, wherein the norbornyl methylenes are located on either the same or the opposite face of the molecule. The stereochemical assignment depicted in Scheme 2 for the major isomer was made in analogy to the observed stereochemistry in subsequent CANAL reactions (vide infra). LAH reduction of $\mathbf{3}$ provides the corresponding tetraol $\mathbf{4}$ with poor solubility in a variety of solvents, limiting our ability to purify it by chromatography. As such, it was taken on crude to the tetra mesylate $(\mathrm{MsCl} / \mathrm{Py})$, which similarly displayed poor solubility in common chromatographic solvents, and was subjected to elimination ( $t$-BuOK / DMPU / DMF) without purification. By this three-step sequence, compound $\mathbf{5}$ was obtained in $38 \%$ overall yield from 3 after purification by flash chromatography.

Tetraene 5 was next subjected to a Diels-Alder cycloaddition $\left(75^{\circ} \mathrm{C}\right)$ with a single equivalent ${ }^{32}$ of quinone dienophile $\mathbf{6}$, the synthesis of which we have previously described. ${ }^{20}$ The reaction is conducted solvent-free under nitrogen, and the crude ${ }^{1} \mathrm{H}$ NMR spectrum shows clean and essentially complete conversion to the single addition product, with none of the bis-cycloaddition product detected. ${ }^{33}$ This crude material was subjected to aromatization to compound 7 using protocols known to be effective in our synthesis of TIPS$\mathrm{BP} 1^{\prime}$. These conditions consist of subjection to basic alumina in toluene with rapid stirring and exposure to air. While this procedure is effective for the synthesis of a variety of norbornyl-bridged acene quinones, here it provides mostly decomposed material, with a small quantity of the desired aromatized cycloadduct. Other aromatization reagents and conditions were studied on related systems without success. These include the use of silica (no reaction at RT); alumina embedded with potassium permanganate ${ }^{34}$ (decomposition of the starting material with no product recovered at temperatures as low as $-32{ }^{\circ} \mathrm{C}$ ); and DDQ or $p$-chloranil (no reaction at room temp, cycloaddition with the terminal diene, with no desired dehydrogenation observed at elevated temperature). Though we had limited supplies of product 7, we pushed our synthesis forward with the amounts we were able to produce from the alumina oxidation conditions. Subjection of 7 to Diels-Alder cycloaddition with quinone $\mathbf{6}$ was performed using conditions akin to the first cycloaddition (neat, $90^{\circ} \mathrm{C}$ ) and similarly produced clean cycloadduct in essentially quantitative mass balance. Unfortunately, aromatization of the 
Diels-Alder product to $\mathbf{8}$ again proved problematic with no conditions providing useable amounts of material.

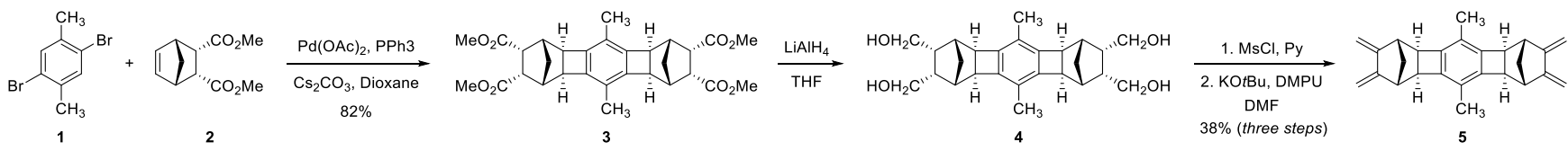

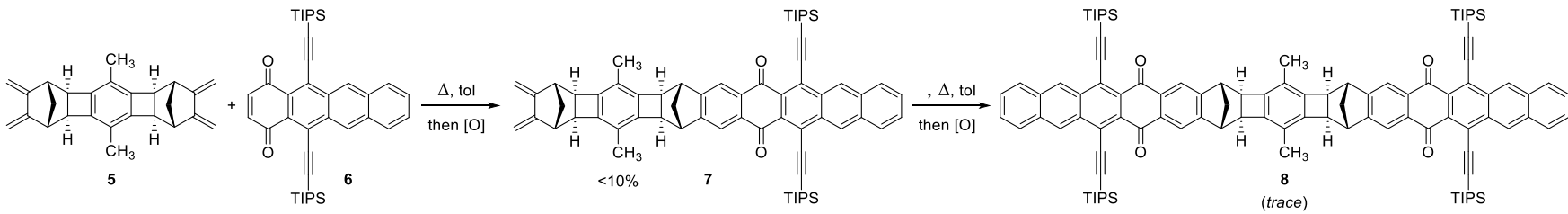

Scheme 2. Initial approach to TIPS-BPX.

The reticence of these Diels-Alder cycloadducts to undergo oxidative aromatization in comparison to our previous studies suggests that the cyclobutene moieties play a detrimental role, providing a pathway for decomposition in preference to aromatization. ${ }^{35,36}$ We note that cyclobutenyl moieties are not present in the successful oxidative aromatization reactions required for the synthesis of TIPSBT1' and TIPS-BP1' and we speculate that cyclobutyl carbinyl radical species are formed in the aromatization pathway and that these undergo fragmentation to provide complex mixtures of products. ${ }^{18,20}$

BTX; A Second Target and Approach. Given the challenges discussed above for TIPS-BPX, a revised strategy was pursued and explored in the synthesis of a tetracene dimer analogue (TIPS-BTX as opposed to TIPS-BPX). We chose the tetracene variant as we reasoned that smaller and more soluble species would provide for more facile reactions and greater opportunity to optimize reaction conditions. Our approach was still transform-goal driven with the use of the CANAL reaction to construct the cyclobutenyl rings, but we wished to employ this transformation later in the synthesis after application of the oxidative aromatization which is problematic in the presence of the cyclobutenyl groups. Our retrosynthetic disconnection is shown in Scheme 3 and proceeds via dibromide 1 and norbornene 11. Application of the CANAL reaction to these substrates would give a bis-quinone product that upon reductive aromatization would provide the target compounds.<smiles>Cc1cc(Br)c(C)cc1Br</smiles>

Scheme 3. Retrosynthesis of TIPS-BTX.

In the forward direction (Scheme 4), our synthesis begins with the known ${ }^{37}$ triene 9 , the preparation of which can be accomplished in two steps from cyclopentadiene. Subjecting 9 to a Diels-Alder reaction with dienophile $\mathbf{1 0}^{20}$ followed by aromatization (alumina / air, RT) provides cycloadduct 11 in $56 \%$ yield without the decomposition issues observed in the preparation of 7 . Our initial attempts at application of the CANAL reaction of dibromo- $p$-xylene derivative $\mathbf{1}$ with olefin $\mathbf{1 1}$, a much larger coupling partner than its analogue 
(2) in our initial synthesis, yielded primarily the mono-coupling product (17\% yield) with a small quantity of the desired bisquinone (12, $8 \%$ yield). Fortunately, the use of JohnPhos in place of triphenylphosphine $\left(\mathrm{Pd}(\mathrm{OAc})_{2} / \mathrm{JohnPhos}{ }^{38,39} / \mathrm{Cs}_{2} \mathrm{CO}_{3}\right)$ provided bisquinone $\mathbf{1 2}$ in 59\% yield, with little observed mono-coupling product. Similar trends in reactivity have been observed by Xia. ${ }^{38,39}$

This transformation, which is the penultimate step of the synthesis, can, in principle, provide up to 6 diastereomers, but because of the high exo-selectivity of the CANAL reaction, ${ }^{31,39}$ only two diastereomers are formed. Interestingly, these are produced in a 13:1 ratio and are separable by flash chromatography. The major isomer was crystallized from toluene, and its structure determined by Xray crystallography (Figure 2). This was found to be the syn-bis-tetracene-quinone (12), with the anti-bis-tetracene-quinone (14) as the minor diastereoisomer. Remarkable in this transformation is not only the levels of remote stereoselection, but also that the seemingly contra-steric product with the two norbornyl methylenes on the same side of the molecule is major.

Reduction of syn-bis-tetracene-quinone $12\left(\mathrm{NaBH}_{4} / \mathrm{DCM} / \mathrm{MeOH}\right)$ provides tetraol intermediates, which were subjected without purification to reductive aromatization $\left(\mathrm{SnCl}_{2} / \mathrm{THF}\right)$ to provide acene dimer TIPS-BTX (13) in $52 \%$ yield. We note an improvement in this reaction using this process wherein we isolate the crude tetraol vs our previous one-pot approach and speculate that this is due to the superior solubility of stannous chloride in THF as opposed to the mixed solvent system of the one-pot procedure. The final target (13, TIPS-BTX) is sensitive to untreated silica, but not to silica deactivated by prior washing with triethylamine. We speculate that the acidity of silica promotes decomposition via protonation of the acene concomitant with opening of the cyclobutyl moiety.
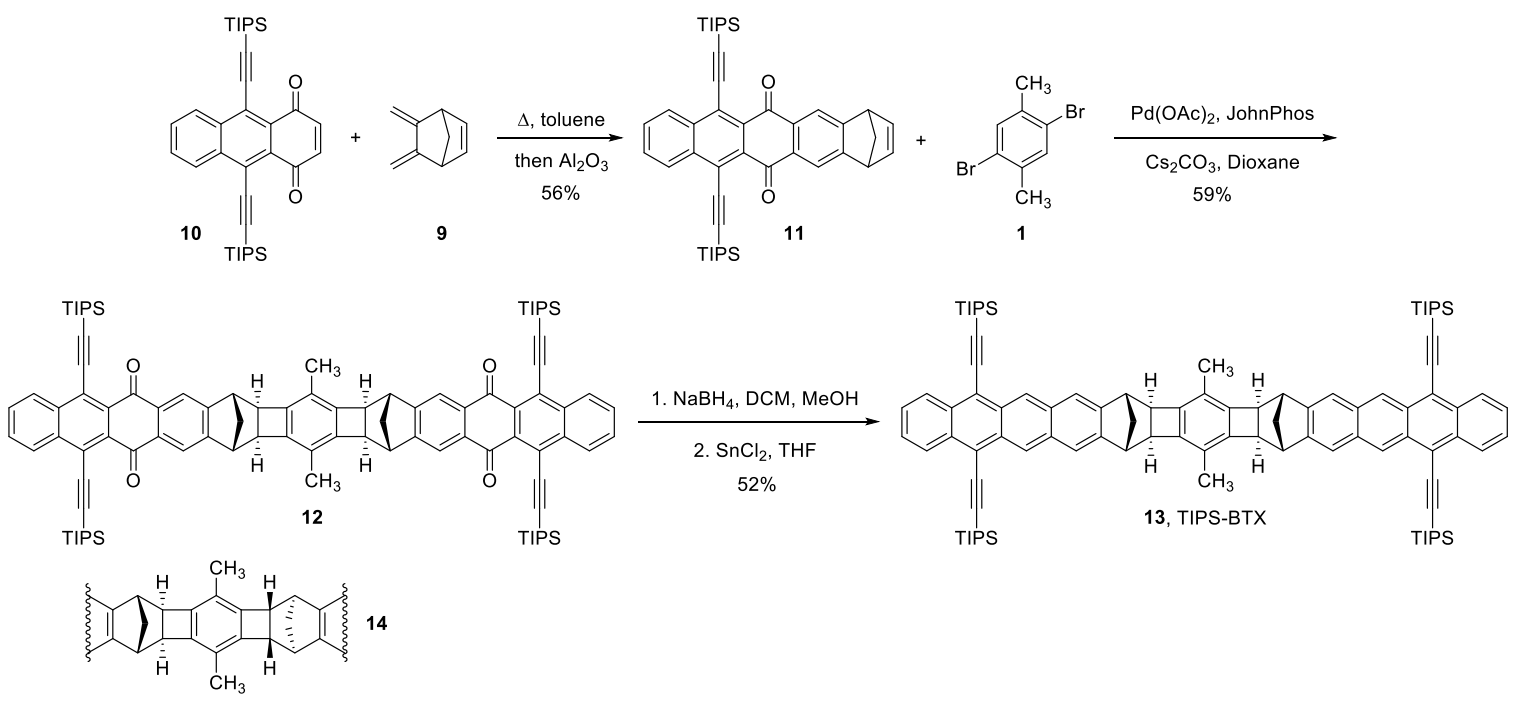

Scheme 4. Synthesis of TIPS-BTX. 


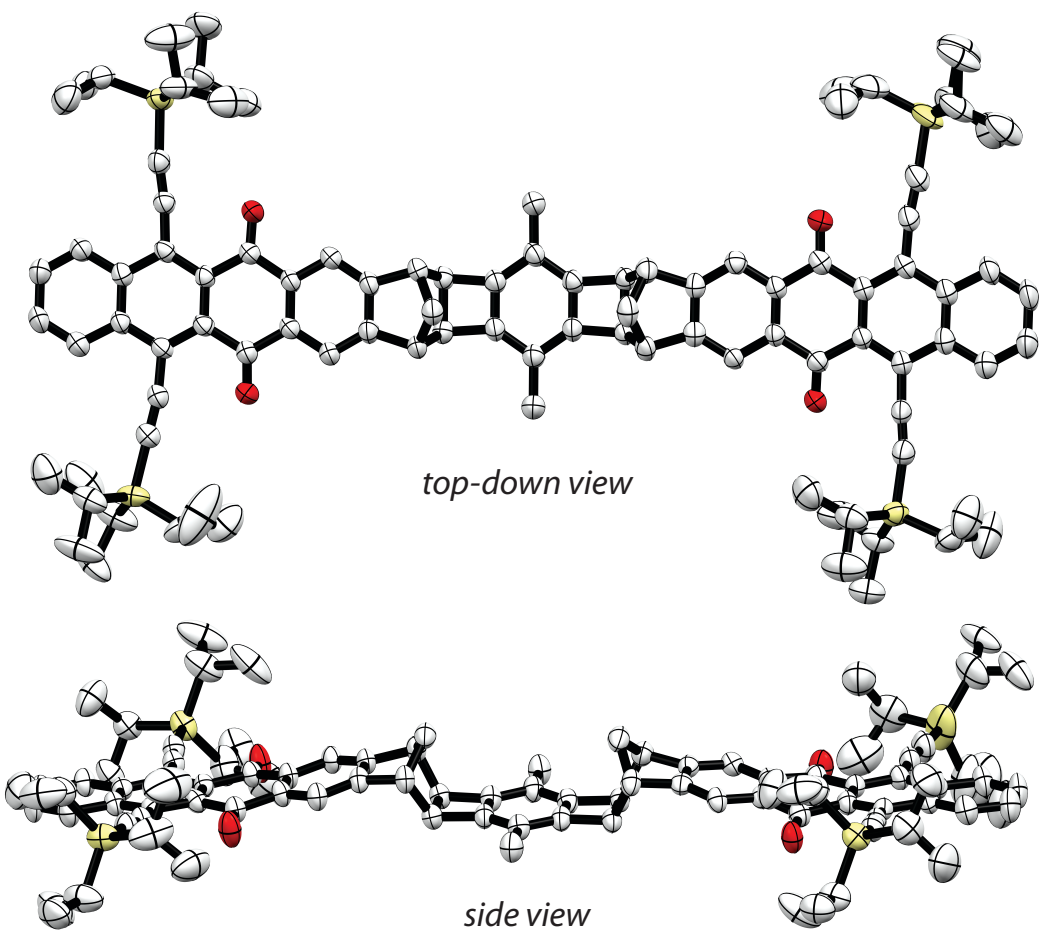

Figure 2. Crystal structure of syn-bis-tetracene-quinone 12 from two perspectives with thermal ellipsoids rendered at $50 \%$ probability. $\mathrm{C}=$ white, $\mathrm{O}=$ red, $\mathrm{Si}=$ yellow. Hydrogen atoms have been removed for clarity. As described in the S.I. but not shown here (again for clarity), there are $\sim 4$ disordered toluene solvent molecules as well as disorder in two of the four TIPS substituents.

This synthesis illustrates the use of the CANAL reaction for the preparation of novel acene dimers. Our transition from a longer, iterative cycloaddition strategy to a two-directional/one-pot strategy not only shortens the synthesis, but also highlights the importance of considering functional group sensitivity in synthetic planning. An important lesson learned from this work then is that careful consideration of the timing of the oxidative aromatization in conjunction with the CANAL reaction avoids the formation of undesired sideproducts due to the presence of sensitive moieties. This work also demonstrates the expansive scope of the CANAL reaction and may render future targets more accessible. The synthetic strategies developed are now being employed to generate the pentacene-derived analogue TIPS-BPX, which will have a larger driving force for the formation of multiexciton states which we predict will be longerlived.

Acknowledgements. This work was funded by the United States Department of Energy, Office of Basic Energy Sciences (ERW7404). The authors thank Laura Maurer of CU Boulder for her initial efforts to collect a suitable single crystal x-ray diffraction data set.

(1) Hanna, M. C.; Nozik, A. J. Solar Conversion Efficiency of Photovoltaic and Photoelectrolysis Cells with Carrier Multiplication Absorbers J. Appl. Phys. 2006, 100, 074510. 
(2) Dexter, D. L. Two Ideas on Energy-Transfer Phenomena - Ion-Pair Effects Involving the OH Stretching Mode, and Sensitization of Photo-Voltaic Cells J. Lumin. 1979, 18-9, 779-784.

(3) Smith, M. B.; Michl, J. Singlet Fission Chem. Rev. 2010, 110, 6891-6936.

(4) Müller, A. M.; Avlasevich, Y. S.; Schoeller, W. W.; Müllen, K.; Bardeen, C. J. Exciton Fission and Fusion in Bis(Tetracene) Molecules with Different Covalent Linker Structures. J. Am. Chem. Soc. 2007, 129, 14240-14250.

(5) Johnson, J. C.; Akdag, A.; Zamadar, M.; Chen, X.; Schwerin, A. F.; Paci, I.; Smith, M. B.; Havlas, Z.; Miller, J. R.; Ratner, M. A.; Nozik, A. J.; Michl, J. Toward Designed Singlet Fission: Solution Photophysics of Two Indirectly Coupled Covalent Dimers of 1,3-Diphenylisobenzofuran J. Phys. Chem. B 2013, 117, 4680-4695.

(6) Sanders, S. N.; Kumarasamy, E.; Pun, A. B.; Trinh, M. T.; Choi, B.; Xia, J. L.; Taffet, E. J.; Low, J. Z.; Miller, J. R.; Roy, X.; Zhu, X. Y.; Steigerwald, M. L.; Sfeir, M. Y.; Campos, L. M. Quantitative Intramolecular Singlet Fission in Bipentacenes J. Am. Chem. Soc. 2015, 137, 8965-8972.

(7) Lukman, S.; Musser, A. J.; Chen, K.; Athanasopoulos, S.; Yong, C. K.; Zeng, Z. B.; Ye, Q.; Chi, C. Y.; Hodgkiss, J. M.; Wu, J. S.; Friend, R. H.; Greenham, N. C. Tuneable Singlet Exciton Fission and Triplet-Triplet Annihilation in an Orthogonal Pentacene Dimer Adv. Funct. Mater. 2015, 25, 5452-5461.

(8) Zirzlmeier, J.; Lehnherr, D.; Coto, P. B.; Chernick, E. T.; Casillas, R.; Basel, B. S.; Thoss, M.; Tykwinski, R. R.; Guldi, D. M. Singlet Fission in Pentacene Dimers Proc. Natl. Acad. Sci. USA 2015, 112, 5325-5330.

(9) Korovina, N. V.; Das, S.; Nett, Z.; Feng, X.; Joy, J.; Haiges, R.; Krylov, A. I.; Bradforth, S. E.; Thompson, M. E. Singlet Fission in a Covalently Linked Cofacial Alkynyltetracene Dimer J. Am. Chem. Soc. 2016, 138, 617-627.

(10) Sakuma, T.; Sakai, H.; Araki, Y.; Mori, T.; Wada, T.; Tkachenko, N. V.; Hasobe, T. Long-Lived Triplet Excited States of Bent-Shaped Pentacene Dimers by Intramolecular Singlet Fission J. Phys. Chem. A 2016, 120, 1867-1875.

(11) Margulies, E. A.; Miller, C. E.; Wu, Y.; Ma, L.; Schatz, G. C.; Young, R. M.; Wasielewski, M. R. Enabling Singlet Fission by Controlling Intramolecular Charge Transfer in $\pi$-Stacked Covalent Terrylenediimide Dimers Nat. Chem. 2016, 8, 1120-1125.

(12) Kumarasamy, E.; Sanders, S. N.; Tayebjee, M. J. Y.; Asadpoordarvish, A.; Hele, T. J. H.; Feummeler, E. G.; Pun, A. B.; Yablon, L. M.; Low, J. Z.; Paley, D. W.; Dean, J. C.; Choi, B.; Scholes, G. D.; Steigerwald, M.; Ananth, N.; McCamey, D. R.; Sfeir, M. Y.; Campos, L. M. Tuning Singlet Fission in Pi-Bridge-Pi Chromophores J. Am. Chem. Soc 2017, 139, 12488-12494.

(13) Yamakado, T.; Takahashi, S.; Watanabe, K.; Matsumoto, Y.; Osuka, A.; Saito, S. Conformational Planarization versus Singlet Fission: Distinct Excited-State Dynamics of Cyclooctatetraene-Fused Acene Dimers Angew. Chem. Int. Edit. Eng. 2018, 57, 5438-5443.

(14) Vallett, P. J.; Snyder, J. L.; Damrauer, N. H. Tunable Electronic Coupling and Driving Force in Structurally WellDefined Tetracene Dimers for Molecular Singlet Fission: A Computational Exploration Using Density Functional Theory J. Phys. Chem. A 2013, 117, 10824-10838.

(15) Alguire, E. C.; Subotnik, J. E.; Damrauer, N. H. Exploring Non-Condon Effects in a Covalent Tetracene Dimer: How Important Are Vibrations in Determining the Electronic Coupling for Singlet Fission? J. Phys. Chem. A 2015, 119, 299-311.

(16) Damrauer, N. H.; Snyder, J. L. Symmetry-Directed Control of Electronic Coupling for Singlet Fission in Covalent BisAcene Dimers J. Phys. Chem. Lett. 2015, 6, 4456-4462.

(17) Cook, J.; Carey, T. J.; Damrauer, N. H. Solution-Phase Singlet Fission in a Structurally Well-Defined NorbornylBridged Tetracene Dimer J. Phys. Chem. A 2016, 120, 4473-4481.

(18) Carey, T. J.; Snyder, J. L.; Miller, E. G.; Sammakia, T.; Damrauer, N. H. Synthesis of Geometrically Well-Defined Covalent Acene Dimers for Mechanistic Exploration of Singlet Fission J. Org. Chem. 2017, 82, 4866-4874.

(19) Cook, J. D.; Carey, T. J.; Arias, D. H.; Johnson, J. C.; Damrauer, N. H. Solvent-controlled branching of localized versus delocalized singlet exciton states and equilibration with charge transfer in a structurally well-defined tetracene dimer J. Phys. Chem. A 2017, 121, 9229-9242.

(20) Carey, T. J.; Miller, E. G.; Gilligan, A. T.; Sammakia, T.; Damrauer, N. H. Modular Synthesis of Rigid Polyacene Dimers for Singlet Fission Org. Lett. 2018, 20, 457-460.

(21) Gilligan, A. T.; Miller, E. G.; Sammakia, T.; Damrauer, N. H. Using Structurally Well-Defined Norbornyl-Bridged Acene Dimers to Map a Mechanistic Landscape for Correlated Triplet Formation in Singlet Fission J. Am. Chem. Soc. 2019, 141, 5961-5971.

(22) Imperiale, C. J.; Green, P. B.; Miller, E. G.; Damrauer, N. H.; Wilson, M. W. B. Triplet-Fusion Upconversion Using a Rigid Tetracene Homodimer J. Phys. Chem. Lett. 2019, 10, 7463-7469.

(23) Smyser, K. E.; Eaves, J. D. Singlet fission for quantum information and quantum computing: the parallel JDE model Sci.Rep. 2020, 10, 18480.

(24) Divincenzo, D. P. Quantum Computation Science 1995, 270, 255-261.

(25) Doherty, M. W.; Manson, N. B.; Delaney, P.; Jelezko, F.; Wrachtrup, J.; Hollenberg, L. C. L. The nitrogen-vacancy colour centre in diamond Phys. Rep. 2013, 528, 1-45.

(26) Fuchs, G. D.; Dobrovitski, V. V.; Hanson, R.; Batra, A.; Weis, C. D.; Schenkel, T.; Awschalom, D. D. Excited-State Spectroscopy Using Single Spin Manipulation in Diamond Phys. Rev. Lett. 2008, 101, 117601.

(27) Bayliss, S. L.; Laorenza, D. W.; Mintun, P. J.; Kovos, B. D.; Freedman, D. E.; Awschalom, D. D. Optically addressable molecular spins for quantum information processing Science 2020, 370, 1309. 
(28) Weiss, L. R.; Bayliss, S. L.; Kraffert, F.; Thorley, K. J.; Anthony, J. E.; Bittl, R.; Friend, R. H.; Rao, A.; Greenham, N. C.; Behrends, J. Strongly exchange-coupled triplet pairs in an organic semiconductor Nat. Phys. 2017, 13, 176-181.

(29) Tayebjee, M. J. Y.; Sanders, S. N.; Kumarasamy, E.; Campos, L. M.; Sfeir, M. Y.; McCamey, D. R. Quintet Multiexciton Dynamics in Singlet Fission Nat. Phys. 2017, 13, 182-188.

(30) Anslyn, E. V.; Dougherty, D. A. Modern Physical Organic Chemistry; University Science: Sausalito, CA, 2006, p 110112.

(31) Liu, S.; Jin, Z.; Teo, Y. C.; Xia, Y. Efficient Synthesis of Rigid Ladder Polymers via Palladium Catalyzed Annulation J. Am. Chem. Soc. 2014, 136, 17434-17437.

(32) Attempted Diels-Alder reaction with two equivalents of dienophile exclusively provided the single-addition product.

(33) While this is consistent with previous observations wherein [2.2.1]-bicyclo bis-dienes undergo the initial Diels-Alder reaction much more readily than the second (see references 18 and 20), the rational for this is likely distinct for the two systems.

(34) Abdollahi-Alibeik, M.; Mohammadpoor-Baltork, I.; Zolfigol, M. A. Alumina supported potassium permanganate: an efficient reagent for chemoselective dehydrogenation of 2-imidazolines under mild conditions Bioorg. Med.Chem. Lett. 2004, 14, 6079-6082.

(35) Crimmins, M. T.; Dudek, C. M.; Wai-Hing Cheung, A. A fragmentation-rearrangement sequence of cyclobutylcarbinyl radicals Tetrahedron Lett. 1992, 33, 181-184.

(36) Crimmins, M. T.; Wang, Z.; McKerlie, L. A. Rearrangement of cyclobutyl carbinyl radicals: Total synthesis of the spirovetivane phytoalexin ( \pm )-lubiminol Tetrahedron Lett. 1996, 37, 8703-8706.

(37) Bowe, M. A. P.; Miller, R. G. J.; Rose, J. B.; Wood, D. G. M. Preparation and Polymerisation of Some Bicyclic Dienes J. Chem. Soc. 1960, 1541-1547.

(38) Martin, R.; Buchwald, S. L. Palladium-Catalyzed Suzuki-Miyaura Cross-Coupling Reactions Employing Dialkylbiaryl Phosphine Ligands Acc. Chem. Res. 2008, 41, 1461-1473.

(39) Jin, Z.; Teo, Y. C.; Zulaybar, N. G.; Smith, M. D.; Xia, Y. Streamlined Synthesis of Polycyclic Conjugated Hydrocarbons Containing Cyclobutadienoids via C-H Activated Annulation and Aromatization J. Am. Chem. Soc. 2017, 139, 1806-1809. 